\title{
Testing universality of Efimov physics across broad and narrow Feshbach resonances
}

\author{
Jacob Johansen, B. J. DeSalvo, Krutik Patel and Cheng Chin ${ }^{\star}$
}

Efimov physics is a universal phenomenon in quantum threebody systems. For systems with resonant two-body interactions, Efimov predicted an infinite series of three-body bound states with geometric scaling symmetry'. These Efimov states, first observed in cold caesium atoms ${ }^{2}$, have been recently reported in a variety of other atomic systems ${ }^{3-13}$. The intriguing prospect of a universal absolute Efimov resonance position across Feshbach resonances remains an open question. Theories predict a strong dependence on the resonance strength for closed-channel-dominated Feshbach resonances, whereas experimental results have so far been consistent with the universal prediction. Here we directly compare the Efimov spectra in a ${ }^{6} \mathrm{Li}-{ }^{133} \mathrm{Cs}$ mixture near two Feshbach resonances which are very different in their resonance strengths, but otherwise almost identical. Our result shows a clear dependence of the absolute Efimov resonance position on Feshbach resonance strength and a clear departure from the universal prediction for the narrow Feshbach resonance.

The first Efimov resonance position is crucial to understanding Efimov physics, as from it one may derive the absolute scale of parameters for subsequent Efimov resonances. Previous experiments in homonuclear systems yield an interesting observation: the first Efimov resonance position $a_{-}$appears to follow a universal formula $a_{-} \approx-9 r_{\mathrm{vdW}}$ (ref. 14), where $r_{\mathrm{vdw}}$ is the van der Waals length of the molecular potential ${ }^{15}$. Calculations from universal theory based on a single-channel model confirm this 'van der Waals universality' for Feshbach resonances with $s_{\text {res }}>1$ (refs 16-20), where the resonance strength parameter $s_{\text {res }}$ characterizes the strength of the coupling between open and closed channels of the Feshbach resonance ${ }^{15}$. Similar theories also give universal but modified predictions for heteronuclear systems ${ }^{21,22}$. Further calculations predict significant deviations from the universal prediction for resonances with $s_{\text {res }} \ll 1$ (refs 18,23-25). Experiments reaching down to $s_{\text {res }}=0.11$, however, have shown little or no dependence on $s_{\text {res }}$ (ref. 9). Experimental results, including this work, are summarized in Fig. 1.

In this Letter, we compare Efimov spectra in a ${ }^{6} \mathrm{Li}-{ }^{133} \mathrm{Cs}$ mixture near one broad $\left(s_{\text {res }}=0.66\right)$ and one very narrow $\left(s_{\text {res }}=0.05\right)$ interspecies Feshbach resonance at $B_{0}=889$ and $893 \mathrm{G}$, respectively. These two Feshbach resonances, shown in Fig. 2a, differ significantly in resonance strength, but only slightly in Cs-Cs scattering length ( $a_{\mathrm{CsCs}}=200$ and $260 a_{0}$, where $a_{0}$ is the Bohr radius). Therefore, this pair of resonances is an excellent case to assess the influence of the Feshbach resonance strength on Efimov physics while keeping other parameters nearly identical. We observe a significant difference in Efimov resonance position between the two Feshbach resonances. Our measurements also show that the resonance associated with the first Efimov state is suppressed near both Feshbach resonances.

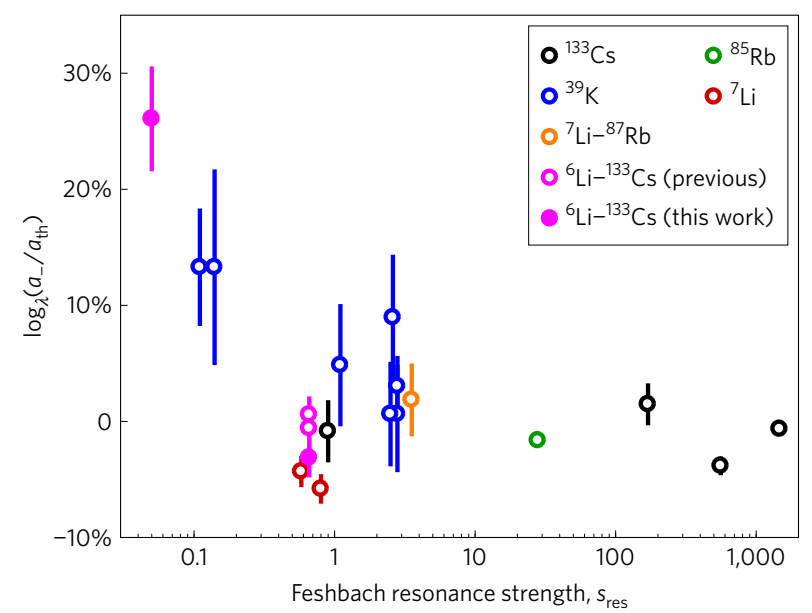

Figure 1 | Deviation of measured first Efimov resonance positions $a_{-}$from predictions based on universal theory $a_{\text {th }}$. For identical bosons,

$a_{\mathrm{th}}=-9.73 \mathrm{r}_{\mathrm{vdW}}$ (ref. 17). For the ${ }^{7} \mathrm{Li}-{ }^{87} \mathrm{Rb}$ Efimov resonance, the universal prediction yields $a_{\mathrm{th}}=-1,800 a_{0}$ (ref. 11 ). $\ln { }^{6} \mathrm{Li}-{ }^{133} \mathrm{Cs}, a_{\mathrm{th}}=-320 a_{0}$ for the $843 \mathrm{G}$ resonance, $a_{\mathrm{th}}=-2,150 a_{0}$ for the $889 \mathrm{G}$ resonance, extracted from ref. 28 , and $a_{\text {th }}=-2,200 a_{0}$ for the $893 \mathrm{G}$ resonance (see text). To compare systems with different Efimov periods $\lambda$, fractional deviations are shown. Previous measurements are plotted as open circles: ${ }^{133} \mathrm{Cs}$ (ref. 14) (black), ${ }^{7} \mathrm{Li}$ (refs 3,5) (red), ${ }^{39} \mathrm{~K}$ (ref. 9) (blue), ${ }^{85} \mathrm{Rb}$ (ref. 8) (green), ${ }^{7} \mathrm{Li}-{ }^{87} \mathrm{Rb}$ (ref. 11) (orange), and ${ }^{6} \mathrm{Li}-{ }^{133} \mathrm{Cs}$ (refs $12,13,28$ ) (magenta). Data from this work are shown as closed circles. Error bars correspond to the one standard deviation total uncertainty.

To resolve Efimov features near the narrow Feshbach resonance at $893 \mathrm{G}$, we require milligauss level control of our magnetic field. For this, we improve our magnetic field control from our previous work $^{12}$. In addition, we develop a scheme to precisely monitor the magnetic field, achieving a precision below $3 \mathrm{mG}$ in each measurement (see Methods). We also incorporate a new dual colour optical trap to eliminate relative gravitational sag of the atoms, which prevented mixing of the two species at temperatures below $200 \mathrm{nK}$ in our previous work ${ }^{12}$ (see Methods).

With these improvements to the apparatus, we prepare spinpolarized $\mathrm{Li}$ and Cs samples at temperatures as low as $50 \mathrm{nK}$ with tunable overlap. We vary magnetic field to tune the interspecies scattering length. In each scan, we randomly order the magnetic fields to eliminate systematic drifts; we also choose an interaction time and overlap to optimize the sensitivity to the collision rate. Details regarding the experimental preparation are provided in the Methods.

The James Franck Institute, Enrico Fermi Institute, and Department of Physics, The University of Chicago, Chicago, Illinois 60637, USA. 

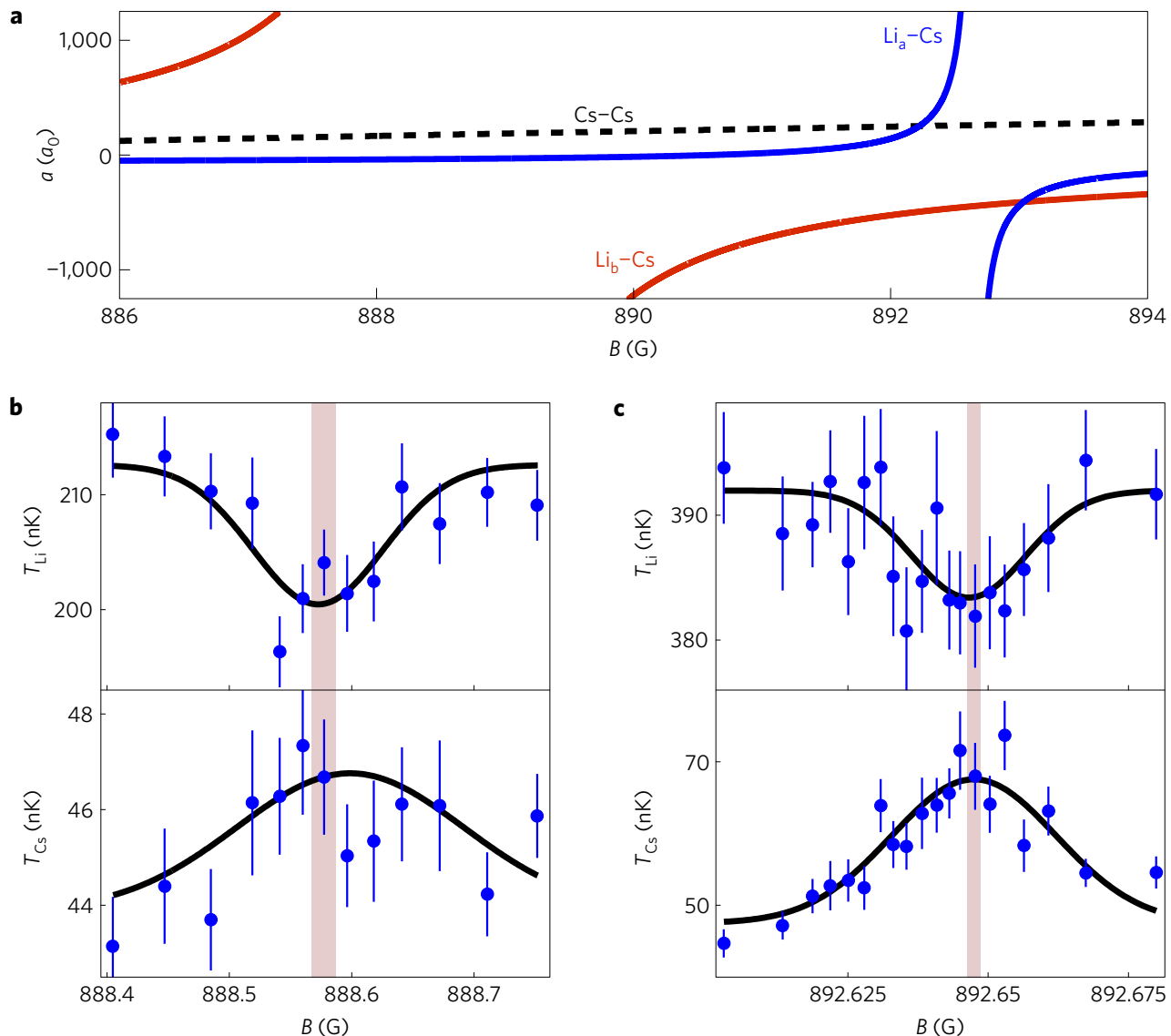

Figure $\mathbf{2}$ | Feshbach resonances employed in this work. a, Calculated scattering lengths as a function of magnetic field in the region of interest, showing a narrow resonance in the $\mathrm{Li}_{\mathrm{a}}-\mathrm{Cs}$ spin channel (blue line) and a broad resonance in the $\mathrm{Li}_{\mathrm{b}}-\mathrm{Cs}$ spin channel (red line) ${ }^{26}$. The Cs-Cs scattering length is shown as a dashed line. b, c, Cross-thermalization between Li and Cs across the Feshbach resonances after interaction times of 200 and $250 \mathrm{~ms}$, respectively. Gaussian fits yield positions of $888.577(10)(10)$ and $892.648(1)(10) \mathrm{G}$, respectively. The shaded region on each plot indicates the one standard deviation statistical uncertainty in Feshbach resonance position, determined from the variance-weighted average of the temperature fits (black curves), while the error bars indicate the $1 \sigma$ standard error of the mean for individual data points.

To determine the interspecies scattering length $a$ at which features occur, we require precise knowledge of Feshbach resonance positions. Previous work based on three-body loss and radiofrequency spectroscopy ${ }^{26,27}$ offers a model to extract the scattering length from the magnetic field; however, higher magnetic field precision is needed for analysis near the narrow resonance. We develop a procedure to precisely determine the resonance positions based on cross-species thermalization. We first create a significant temperature difference between $\mathrm{Li}$ and Cs samples, with $T_{\mathrm{Cs}} \approx 45 \mathrm{nK}$ and $T_{\mathrm{Li}} \approx 300 \mathrm{nK}$. After the interaction time, we measure the final temperatures. Near an interspecies Feshbach resonance, enhanced elastic collisions between the two species lead to enhanced interspecies thermalization. This approach has a significant advantage over measurements relying on three-body recombination or radio-frequency spectroscopy because the elastic collision rate is symmetric about the resonance over the small range we probe and does not depend on a complex model to extract the resonance position ${ }^{15}$.

The interspecies thermalization measurements are shown in Fig. 2. We determine the resonance positions with a Gaussian fit to the measured temperatures. The extracted resonance positions from $\mathrm{Li}$ and Cs data agree within the uncertainty. The varianceweighted resonance positions are $B_{\text {broad }}=888.577(10)(10) \mathrm{G}$ and $B_{\text {narrow }}=892.648(1)(10) \mathrm{G}$, where the values in parentheses indicate the statistical and systematic uncertainties, respectively. Notably, the systematic uncertainty is dominated by factors which are common to all measurements. As a result, magnetic field offsets relative to the Feshbach resonance positions, $\Delta B_{\mathrm{b}}$ and $\Delta B_{\mathrm{n}}$, have a reduced systematic uncertainty (see Methods). Our results are consistent with refs 26,27 while offering much higher precision.

To see Efimov features near these Feshbach resonances, we modify the experimental sequence to optimize the three-body loss signal. We prepare $\mathrm{Li}$ and $\mathrm{Cs}$ at nearly equal temperatures of approximately $100 \mathrm{nK}$. After the interaction time, we measure Li and Cs atom numbers by absorption imaging (see Methods).

The atomic loss spectra are shown in Fig. 3. Coarse scans across the Feshbach resonances provide context for the Efimov features (see Fig. 3a,e). Finer scans at negative scattering length, shown in Fig. 3c,g, display enhanced loss features, identified as Efimov resonances. These features are qualitatively similar, but with different magnetic field scales. Using a Gaussian fit with a linear background, we determine the Efimov resonance positions to be $0.842(12)(10)$ and 0.041(1)(2) G above the broad and narrow Feshbach resonance, respectively. Using the model in ref. 27 and our measurements of the Feshbach resonance positions, we calculate the corresponding scattering lengths as $a=-2,050(60)$ and $-3,330(240) a_{0}$ for the broad and narrow resonance, respectively. The quoted uncertainties in scattering length include the previously discussed statistical and systematic uncertainties in magnetic field, as well as an additional $2 \%$ uncertainty in the model $^{27}$.

Comparing with our previous measurement near the broad Feshbach resonance at $843 \mathrm{G}$ (ref. 12), where the first Efimov resonance occurs at $-323(8) a_{0}$, both Efimov resonance positions in the present work are more than a full Efimov period higher in magnitude. This suggests the existence of a lower-order Efimov 
a
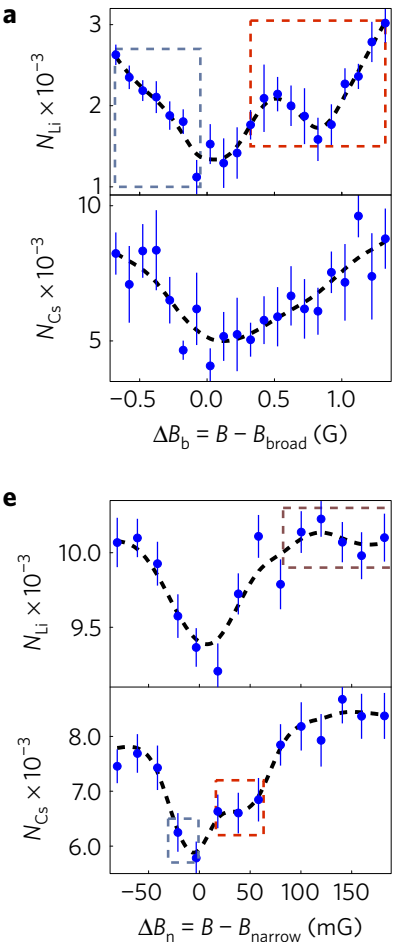

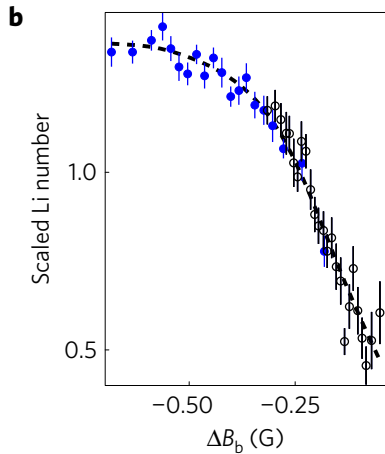

$\mathbf{f}$

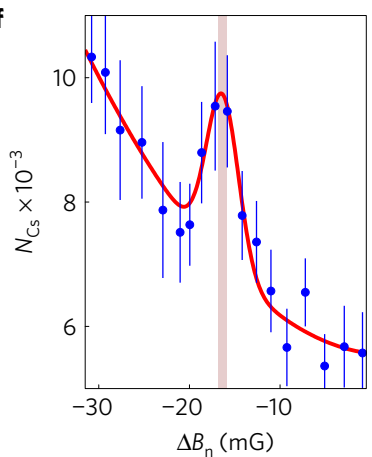

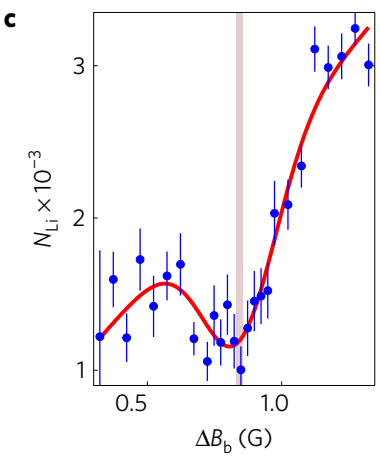

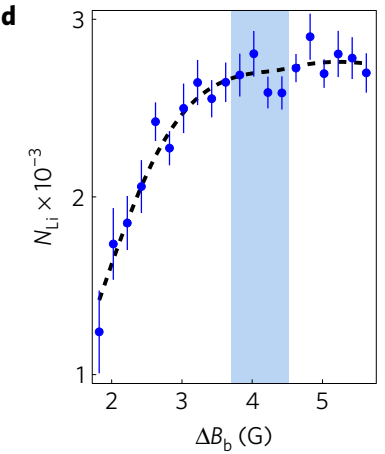

g

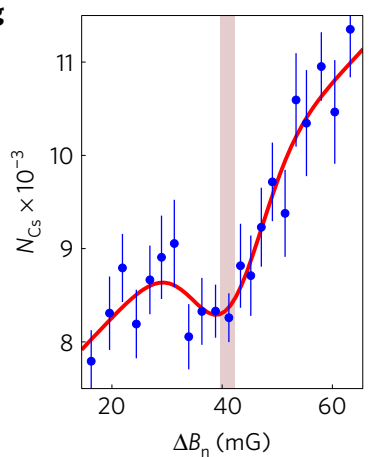

h

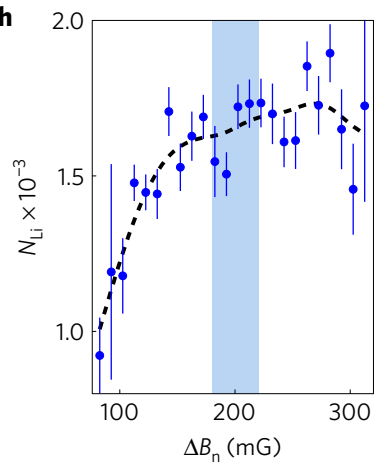

Figure 3 | Measurement of Efimov features near the broad and narrow Feshbach resonances. All magnetic fields are plotted relative to the Feshbach resonance positions determined in Fig. 2. a-d, Features near the broad resonance. e-h, Features near the narrow resonance. a,e, Broad scans across the Feshbach resonances. Grey, red, and brown boxes indicate the regions of the fine scans shown in $\mathbf{b}$ and $\mathbf{f}$, $\mathbf{c}$ and $\mathbf{g}$, and $\mathbf{h}$, respectively. $\mathbf{b}, \mathbf{f}$, Scans on the positive scattering length side of Feshbach resonances. Near the narrow resonance ( $\mathbf{f})$, we identify suppression of three-body loss at $\Delta B_{\mathrm{n}}=-16(1)(2) \mathrm{mG}$. Two scans are shown in $\mathbf{b}$. Since the scans are performed under different conditions we scale the data to the average value in the overlapping region, clarifying the overall trend. $\mathbf{c}, \mathbf{g}$, Observation of an Efimov resonance on the negative side of each Feshbach resonance, at $\Delta B_{\mathrm{b}}=842(12)(10) \mathrm{mG}(\mathbf{c})$ and at $\Delta B_{\mathrm{n}}=41(1)(2) \mathrm{mG}(\mathbf{g}) . \mathbf{d}, \mathbf{h}$, Scans in the range expected for the lower-order Efimov resonance; the blue shaded regions indicate the expected positions

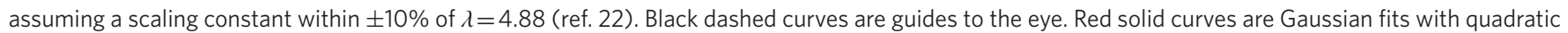
(in f) or linear (in all other cases) backgrounds used to extract positions of features, while the corresponding red shaded regions indicate one standard deviation statistical uncertainty. Error bars indicate the $1 \sigma$ standard error.

state. However, searching in the ranges expected from geometric scaling ${ }^{12}$, we do not observe any additional Efimov resonances (see Fig. 3d,h). The missing feature has been studied for the resonance at $889 \mathrm{G}$ (ref. 28) and arises from a weakly bound $\mathrm{Cs}_{2}$ state, which suppresses the resonance due to the ground Efimov state. We find that the first Efimov resonance is missing for both the broad and narrow Feshbach resonances measured here. Based on this interpretation, the Efimov resonances observed in this work stem from the second Efimov states.

Additionally, we observe suppression of three-body loss at positive interspecies scattering length (see Fig. $3 \mathrm{f}$ and Supplementary Information). The suppression occurs at $\Delta B_{\mathrm{n}}=-16(1)(2) \mathrm{mG}$, corresponding to $a=8,100(1,300) a_{0}$. Searching for a similar feature near the broad Feshbach resonance over the range 887.9 to $888.5 \mathrm{G}$ ( $a=2,500$ to $30,000 a_{0}$ ), however, yields no discernible features (see Fig. 3b).

We present the results of all scans in terms of interspecies scattering length in Fig. 4 to compare Efimov features near the broad and narrow Feshbach resonances. To combine all scans, we convert atom number to the three-body recombination coefficient $K_{3}$ based on a numerical model of three-body loss described in the Methods. Owing to uncertainties in trapping frequencies and overlap between the Li and Cs clouds, we scale $K_{3}$ to the measured value far from resonance $K_{3}^{(0)}$ (shown in Fig. 4 insets). From this figure, we can clearly see a difference in the Efimov resonance positions for the broad and narrow Feshbach resonances, indicated by the solid arrows. We can also see significant deviation from the universal prediction (magenta dotted lines) for the narrow Feshbach resonance.
For precise quantitative comparison of Efimov resonances, as well as comparison to the universal theory, we summarize Feshbach and Efimov resonance positions in ${ }^{6} \mathrm{Li}-{ }^{133} \mathrm{Cs}$ in Table 1 . Due to the suppressed resonance from the first Efimov states, discussed above, we compare resonance positions from the second Efimov states, which we label $a_{-}^{(2)}$. Between the broad and narrow Feshbach resonances near $890 \mathrm{G}$, we observe a large difference of $63(12) \%$ in $a_{-}^{(2)}$, while the universal theory predicts a much smaller effect of $2.3 \%\left(-2,150 a_{0}\right.$ for the broad resonance, extracted from ref. 28 , and $-2,200 a_{0}$ for the narrow resonance, calculated using the same method, outlined in ref. 29). This difference is expected from the small change in $a_{\mathrm{CsCs}}$. Furthermore, the universal prediction is in excellent agreement with measurements for both broad resonances at 843 and $889 \mathrm{G}$, in spite of the significant difference in $a_{\mathrm{CsCs}}$. This agreement indicates that the universal theory describes broad resonances. However, it does not capture the behaviour of the narrow resonance, as our measurement near the narrow resonance at $893 \mathrm{G}$ deviates from the universal theory by $51(11) \%$.

Placing this work in the broader context of previous studies offers insight into the universality of Efimov physics in cold atom systems. Based on the general form $K_{3} \propto\left\{\sin ^{2}\left[\pi \log _{\lambda}\left(a / a_{-}\right)\right]+\text {const. }\right\}^{-1}$ for $a<0$ (ref. 30), we introduce a phase shift $\Delta \Phi=\pi \log _{\lambda}\left(a_{-} / a_{\mathrm{th}}\right)$ which characterizes deviation from the universal prediction and accounts for the scaling constant $\lambda$. As such, the metric $\Delta \Phi / \pi$ can be used to compare our result to other experiments with different scaling constants (as shown in Fig. 1). Our result at $s_{\text {res }}=0.05$, the smallest resonance strength thus far investigated, offers a clear deviation of 26(5)\% of an Efimov period from the universal 

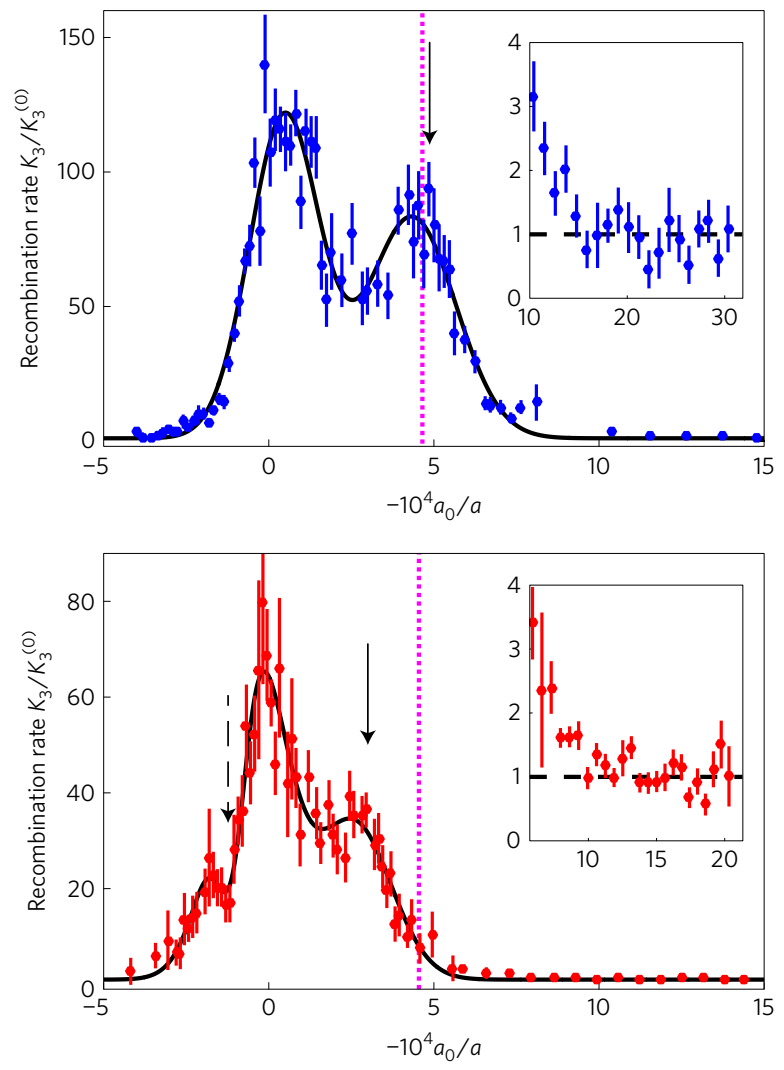

Figure 4 | Recombination rate near the broad and narrow Feshbach resonances. a,b, Recombination rate $K_{3}$ is plotted as a function of the inverse scattering length for the broad resonance near $889 \mathrm{G}(\mathbf{a})$ and the narrow resonance near $893 \mathrm{G}$ (b). The recombination rate is normalized to the off-resonant value $K_{3}^{(0)}$, see dashed lines in the insets. Vertical solid arrows indicate Efimov resonance positions. The dashed arrow in $\mathbf{b}$ indicates a suppression feature in the three-body loss rate at positive scattering length near the narrow resonance. The magenta dotted lines indicate the positions predicted by the universal theory, while black curves are guides to the eye consisting of the sum of Gaussians. Error bars indicate the $1 \sigma$ standard error.

Table 1 | Summary of Efimov resonances for the three Feshbach resonances in Li-Cs at 843 (ref. 12), 889, and $893 \mathrm{G}$.

\begin{tabular}{lllll}
$B_{0}(G)$ & $s_{\text {res }}$ & $a_{\mathrm{CsCs}\left(a_{0}\right)}$ & $\boldsymbol{a}_{-}^{(2)}\left(a_{0}\right)$ & $a_{\mathrm{th}}^{(2)}\left(a_{0}\right)$ \\
\hline $888.577(10)(10)$ & 0.66 & 200 & $-2,050(60)$ & $-2,150$ \\
$892.648(1)(10)$ & 0.05 & 260 & $-3,330(240)$ & $-2,200$ \\
$842.750(1)(3)$ & 0.66 & $-1,400$ & $-1,635(60)$ & $-1,680$ \\
\hline
\end{tabular}

Here, $B_{0}$ indicates the Feshbach resonance position, $s_{\text {res }}$ the Feshbach resonance strength, $a_{\mathrm{CsCs}}$ the Cs-Cs scattering length, $a_{-}^{(2)}$ the resonance position associated with the second Efimov state, and $a_{\mathrm{th}}^{(2)}$ the prediction from the universal theory using a single-channel model $^{28,29}$

prediction (a 5 standard deviation effect). As published theories describing narrow resonances are constrained to homonuclear systems, deeper understanding of the Efimov resonance dependence on $s_{\text {res }}$ will require theoretical work tailored to heteronuclear systems beyond single-channel calculations. Nevertheless, our result already suggests a trend of increasing Efimov resonance position with decreasing $s_{\text {res }}$.

\section{Methods}

Methods, including statements of data availability and any associated accession codes and references, are available in the online version of this paper.
Received 27 December 2016; accepted 10 April 2017;

published online 15 May 2017; corrected online 23 May 2017

\section{References}

1. Efimov, V. Energy levels arising from resonant two-body forces in a three-body system. Phys. Lett. B 33, 563-564 (1970).

2. Kraemer, T. et al. Evidence for Efimov quantum states in an ultracold gas of caesium atoms. Nature 440, 315-318 (2006).

3. Pollack, S. E., Dries, D. \& Hulet, R. G. Universality in three- and four-body bound states of ultracold atoms. Science 326, 1683-1685 (2009).

4. Zaccanti, M. et al. Observation of an Efimov spectrum in an atomic system. Nat. Phys. 5, 586-591 (2009).

5. Gross, N., Shotan, Z., Kokkelmans, S. \& Khaykovich, L. Observation of universality in ultracold ${ }^{7} \mathrm{Li}$ three-body recombination. Phys. Rev. Lett. 103, 163202 (2009)

6. Wenz, A. N. et al. Universal trimer in a three-component Fermi gas. Phys. Rev. A 80, 040702 (2009)

7. Williams, J. R. et al. Evidence for an excited-state Efimov trimer in a three-component Fermi gas. Phys. Rev. Lett. 103, 130404 (2009).

8. Wild, R. J., Makotyn, P., Pino, J. M., Cornell, E. A. \& Jin, D. S. Measurements of Tan's contact in an atomic Bose-Einstein condensate. Phys. Rev. Lett. 108, 145305 (2012)

9. Roy, S. et al. Test of the universality of the three-body Efimov parameter at narrow Feshbach resonances. Phys. Rev. Lett. 111, 053202 (2013).

10. Huang, B., Sidorenkov, L. A., Grimm, R. \& Hutson, J. M. Observation of the second triatomic resonance in Efimov's scenario. Phys. Rev. Lett. 112, 190401 (2014)

11. Maier, R. A. W., Eisele, M., Tiemann, E. \& Zimmermann, C. Efimov resonance and three-body parameter in a lithium-rubidium mixture. Phys. Rev. Lett. 115, 043201 (2015).

12. Tung, S.-K., Jiménez-García, K., Johansen, J., Parker, C. V. \& Chin, C. Geometric scaling of Efimov states in a ${ }^{6} \mathrm{Li}-{ }^{133} \mathrm{Cs}$ mixture. Phys. Rev. Lett. 113, 240402 (2014).

13. Pires, R. et al. Observation of Efimov resonances in a mixture with extreme mass imbalance. Phys. Rev. Lett. 112, 250404 (2014).

14. Berninger, M. et al. Universality of the three-body parameter for Efimov states in ultracold cesium. Phys. Rev. Lett. 107, 120401 (2011).

15. Chin, C., Grimm, R., Julienne, P. \& Tiesinga, E. Feshbach resonances in ultracold gases. Rev. Mod. Phys. 82, 1225 (2010).

16. Chin, C. Universal scaling of Efimov resonance positions in cold atom systems. Preprint at http://arXiv.org/abs/1111.1484 (2011).

17. Wang, J., D'Incao, J. P., Esry, B. D. \& Greene, C. H. Origin of the three-body parameter universality in Efimov physics. Phys. Rev. Lett. 108, 263001 (2012).

18. Schmidt, R., Rath, S. \& Zwerger, W. Efimov physics beyond universality. Eur. Phys. J. B 85, 386-391 (2012).

19. Naidon, P., Endo, S. \& Ueda, M. Microscopic origin and universality classes of the Efimov three-body parameter. Phys. Rev. Lett. 112, 105301 (2014).

20. Naidon, P., Endo, S. \& Ueda, M. Physical origin of the universal three-body parameter in atomic Efimov physics. Phys. Rev. A 90, 022106 (2014).

21. D’Incao, J. P. \& Esry, B. D. Ultracold three-body collisions near overlapping Feshbach resonances. Phys. Rev. Lett. 103, 083202 (2009).

22. Wang, Y., Wang, J., D’Incao, J. P. \& Greene, C. H. Universal three-body parameter in heteronuclear atomic systems. Phys. Rev. Lett. 109, 243201 (2012).

23. Petrov, D. S. Three-boson problem near a narrow Feshbach resonance. Phys. Rev. Lett. 93, 143201 (2004).

24. Gogolin, A. O., Mora, C. \& Egger, R. Analytical solution of the bosonic three-body problem. Phys. Rev. Lett. 100, 140404 (2008).

25. Sørensen, P. K., Fedorov, D. V., Jensen, A. S. \& Zinner, N. T. Efimov physics and the three-body parameter within a two-channel framework. Phys. Rev. A 86, 052516 (2012)

26. Tung, S.-K. et al. Ultracold mixtures of atomic ${ }^{6} \mathrm{Li}$ and ${ }^{133} \mathrm{Cs}$ with tunable interactions. Phys. Rev. A 87, 010702 (2013).

27. Ulmanis, J. et al. Universality of weakly bound dimers and Efimov trimers close to LiCs Feshbach resonances. New J. Phys. 17, 055009 (2015).

28. Ulmanis, J. et al. Heteronuclear Efimov scenario with positive intraspecies scattering length. Phys. Rev. Lett. 117, 153201 (2016).

29. Wang, Y., D’Incao, J. P. \& Esry, B. D. Ultracold few-body systems. Adv. At. Mol. Opt. Phys. 62, 1-115 (2013).

30. D’Incao, J. P. \& Esry, B. D. Scattering length scaling laws for ultracold three-body collisions. Phys. Rev. Lett. 94, 213201 (2005).

\section{Acknowledgements}

We thank Y. Wang, C. Greene and R. Grimm for valuable discussions. We thank L. Feng, C. V. Parker and S.-K. Tung for help in the early stages of the experiment. We 
acknowledge funding support from NSF Materials Research Science and Engineering Centers grant DMR-1420709 and NSF grant PHY-1511696. Additional support for B.J.D is provided by the Grainger Fellowship.

\section{Author contributions}

J.J., B.J.D. and K.P. performed the experiments. C.C. supervised the work. All authors were involved in analysis and discussions of the results and contributed to the preparation of the manuscript.

\section{Additional information}

Supplementary information is available in the online version of the paper. Reprints and permissions information is available online at www.nature.com/reprints. Publisher's note: Springer Nature remains neutral with regard to jurisdictional claims in published maps and institutional affiliations. Correspondence and requests for materials should be addressed to C.C.

\section{Competing financial interests}

The authors declare no competing financial interests. 


\section{Methods}

Dual colour optical dipole trap. In our current work, we use an updated optical dipole trapping scheme. This includes the 1,064 nm elliptical trap and $\sim 1,070 \mathrm{~nm}$ translatable trap discussed in previous work ${ }^{12,26}$ with some minor changes. The elliptical trap is approximately 33 by $350 \mu \mathrm{m}$ at the atomic position, with the tight axis along the vertical direction. As before, it may be translated vertically or modulated to increase its vertical size ${ }^{12}$. However, as in previous work, gravitational sag separates Cs from Li in this trap at very low temperatures.

To circumvent this, we implement a dual colour trap consisting of 1,064 and $785 \mathrm{~nm}$ light co-propagating with the elliptical beam, with maximum powers of 150 and $120 \mathrm{~mW}$, respectively. In our present work, both beams are focused to a waist of $29 \mu \mathrm{m}$ with the $1,064 \mathrm{~nm}$ beam approximately $5 \mu \mathrm{m}$ above the $785 \mathrm{~nm}$ beam. The $785 \mathrm{~nm}$ beam pushes Cs up while pulling down $\mathrm{Li}$, allowing us to counteract the difference in gravitational sag. Using this trap, we may maintain perfect overlap at arbitrarily low temperatures by careful choice of the 785 and $1,064 \mathrm{~nm}$, and elliptical beam powers, as well as the elliptical beam position.

As all of these beams are co-propagating, they only very weakly trap atoms along the propagation (axial) direction. While an additional beam is used to provide axial confinement during initial loading, in the final trap this beam is turned off, and axial confinement is provided by magnetic field curvature with a trapping frequency of approximately $6.5 \mathrm{~Hz}$ for Cs and $36 \mathrm{~Hz}$ for Li near $900 \mathrm{G}$. The use of magnetic trapping ensures that the atoms are located at the centre of curvature of our magnetic field, allowing us to better measure our magnetic field with only small adjustments to our experimental procedure, as detailed below.

Magnetic field calibration. As in our previous work, we use the $\left|F, m_{F}\right\rangle=|3,3\rangle$ to $|4,4\rangle$ (where $F$ and $m_{F}$ denote the total angular momentum and magnetic quantum number, respectively) microwave transition in caesium to calibrate our magnetic field $B$. However, we now use a tomographic approach to measure the field in a single execution of the experimental sequence. We use our ability to move the elliptical beam to measure the field curvature to a precision of approximately $95 \%$. To measure the field, we run a modified version of the sequence, omitting most of the caesium evaporation and leaving caesium at a temperature of $1-2 \mu \mathrm{K}$. We then use a $1 \mathrm{~ms}$ microwave pulse set to a frequency slightly below that which would pump the centre of the cloud, and immediately image the atoms in the $|4,4\rangle$ state. Because of the magnetic field curvature, two regions of the cloud are imaged. The separation between these two imaged regions is determined by the magnetic field curvature, the microwave frequency, and the magnetic field at the centre of the cloud. Because we use magnetic trapping to provide axial confinement, our atoms are located at the centre position and, when fully evaporated, are confined to a very small region around it. Thus, for a fixed microwave frequency, we can determine the magnetic field directly from a single image. Images showing this tomographic technique for magnetic field calibration and a fully evaporated cloud for comparison are contained in the Supplementary Methods.

During normal operation, we use this tomographic technique to characterize our field approximately $5 \mathrm{~s}$ after every shot. This allows us to know our field, in spite of slow drifts in our field on the order of $30 \mathrm{mG}$, with a precision of $3 \mathrm{mG}$ from a single experimental execution. There exists a small but nonzero offset between the calibration and the main experimental sequence, and to control for this offset we precisely measure it each day. No detectable drift between the field calibration and the main experimental sequence exists over the course of a day, as verified from several hundred shots taken over the course of several hours.

The primary systematic uncertainty in the magnetic field is due to the uncertainty in the field curvature. For typical experimental conditions, this contributes approximately $3 \mathrm{mG}$. In addition, temperatures of $100 \mathrm{nK}$ in both lithium and caesium yield a cloud width in the magnetic trap corresponding to approximately $2 \mathrm{mG}$, leading to slight asymmetric broadening of any features narrower than this. Finally, as noted in the previous paragraph, we measure the offset between the field calibration and the main experimental sequence daily, averaging the measurement until the uncertainty is less than $1 \mathrm{mG}$. This calibration uncertainty leads to an additional systematic. Combining all of these systematic uncertainties leads to an estimate of the absolute systematic uncertainty of approximately $4 \mathrm{mG}$. Even with a more conservative estimate of $10 \mathrm{mG}$ which we use in the Letter, our results are not significantly changed.

While systematic errors due to the field curvature influence the absolute magnetic field positions of measured features, they are common to all measurements performed in our system, and therefore do not influence the relative magnetic field positions of these features. For relative magnetic field measurements, the uncertainties due to the magnetic field curvature and the finite temperature effect are negligible. However, the daily offset measurement uncertainty does remain for the relative magnetic field measurement, given by the combined uncertainty of the two uncorrelated offset measurements, leading to a relative systematic uncertainty of $1.4 \mathrm{mG}$. Finally, the Feshbach resonance position statistical uncertainty must be included, leading to a total relative uncertainty of $2 \mathrm{mG}$ for the narrow Feshbach resonance and $10 \mathrm{mG}$ for the broad Feshbach resonance.

Experimental sequence. We load and evaporate Li in the dual colour trap and Cs in the elliptical trap in a manner similar to the procedure described in refs 12,26. As the elliptical trap can be translated vertically, Li and Cs are separated for most of the preparation. Preparation ends as Cs is translated to overlap with Li while the beam powers are gradually changed to allow mixing of $\mathrm{Li}$ and Cs. At the same time, the field is ramped to a value slightly above the Feshbach resonance to be studied: for the narrow resonance, $850 \mathrm{mG}$ away; for the broad resonance, $5.2 \mathrm{G}$ away. In both cases, the absolute value of the scattering length is below $400 a_{0}$, sufficient to suppress interactions during the final stage of preparation.

We then jump the magnetic field to the desired value and hold for an interaction time at fixed magnetic field. The magnetic field is varied from one shot to the next over a fixed range, with the order in which points are taken randomized to eliminate slow drifts. The interaction time is held fixed over each field scan. After the interaction time, the field is jumped back to a fixed value for high-field imaging of Li. We then determine the Li and Cs particle number and temperature using absorption imaging.

The sequence differs slightly for different scans. In particular, the cross-thermalization measurement is performed with increased power in the $785 \mathrm{~nm}$ beam, as this simultaneously increases the Li trap depth and decreases the Cs trap depth, leading to the required temperature imbalance $\left(T_{\mathrm{Li}}=200-400 \mathrm{nK}\right.$, $\left.T_{\mathrm{Cs}}=40-50 \mathrm{nK}\right)$. Furthermore, with the $\mathrm{Li}$ at high temperature, we ensure that it does not form a degenerate Fermi gas, avoiding potential many-body effects which would complicate the cross-thermalization measurement. In addition, during this sequence, the Li and Cs clouds are mostly separated. As a result, the collision rate is reduced, allowing for longer interaction times. This is essential to reduce the importance of the magnetic field settling time of approximately $2 \mathrm{~ms}$. In addition, the three-body recombination rate is suppressed even more than the elastic collision rate by the reduced density in the overlapping region, suppressing three-body loss relative to the cross-thermalization rate.

For the Efimov resonance measurements, a lower $785 \mathrm{~nm}$ beam power is used such that the Li and Cs temperatures are approximately equal, with $T \approx 100 \mathrm{nK}$. However, for measurements very near the Feshbach resonance, we still use partial overlap to slow the three-body recombination rate, reducing the importance of the magnetic field settling time. For measurements far from the Feshbach resonance, with $\mathrm{Li}$-Cs scattering length $|a|<1,000 a_{0}$, we optimize the overlap between the $\mathrm{Li}$ and Cs clouds to compensate for the reduced recombination rate at small scattering length.

After this measurement, we perform a high-field calibration, as detailed above. The total time between the end of the main experimental sequence and the field calibration is $<5 \mathrm{~s}$.

Recombination coefficient $K_{3}$ extraction. As shown in Fig. 3, raw data consists of the measurement of atom number via absorption imaging after a finite interaction time at a specific magnetic field. To compare data across different days and varying conditions, we convert atom loss into a scaled measurement of the three-body recombination coefficient $K_{3}$, which is normalized to the measured value far away from resonance $K_{3}^{(0)}$

To extract the scaled value of $K_{3}$, we adopt a simple model of loss in our system motivated by a number of observations. First, when Li and Cs are not mixed, we measure a lifetime orders of magnitude longer than when they are mixed. As such, we can neglect both three-body loss due to Cs-only collisions and one-body loss. Second, the variation in temperature across each scan is negligible. This allows us to neglect loss due to cross-thermalization and heating atoms out of the trap. With these approximations, and assuming an equilibrium distribution, the atom number of Li and Cs can be modelled with the following set of differential equations

$$
\begin{aligned}
\frac{\mathrm{d} N_{\mathrm{Li}}}{\mathrm{d} t} & =-K_{3} G(T) N_{\mathrm{Cs}}^{2} N_{\mathrm{Li}} \\
\frac{\mathrm{d} N_{\mathrm{Cs}}}{\mathrm{d} t} & =-2 K_{3} G(T) N_{\mathrm{Cs}}^{2} N_{\mathrm{Li}}
\end{aligned}
$$

where $G(T)$ is a time-independent geometric factor accounting for the partial overlap of the clouds given by $G(T)=\left(1 / N_{\mathrm{CS}}^{2} N_{\mathrm{Li}}\right) \int n_{\mathrm{CS}}^{2}(\mathbf{x}, T) n_{\mathrm{Li}}(\mathbf{x}, T) \mathrm{d}^{3} \mathbf{x}$. The factor of two arises from the fact that two Cs atoms are lost in each three-body recombination collision.

As the enhanced (or suppressed) number of atoms lost is small, we find that typically the species with a smaller number of atoms in a given scan exhibits the best signal to noise. Therefore, when we extract $K_{3}$ from a given scan, we fit the number of atoms remaining of whichever species shows the largest signal to noise to the above model. 
Due to slight changes of the trap from day to day, the geometric factor $G(T)$ is difficult to measure to the level of precision necessary. Therefore, we rescale extracted values of $K_{3}$ in each scan such that the average values in regions of overlapping magnetic field match across different scans. Further, for clarity in Fig. 4, data are binned according to inverse interspecies scattering length, and presented as the variance-weighted mean of all data in each bin.

Data availability. The data associated with the plots and this study are available from the corresponding author upon request. 